\title{
SUR UN ASPECT DES ÉTUDES HISTORIQUES
}

En se fondant sur le principe qui dit que la science doit tendre vers le général, on est conduit à établir non seulement des changements concrets mais aussi des tendances évolutives. Nous partageons l'opinion selon laquelle il est possible d'établir des principes généraux de changements linguistiques, définis de différentes façons: principes, tendances, universaux, etc. Les arguments en faveur de cette thèse sont nombreux, ne serait-ce que ceux rappelés par Haspelmath (1999: 87). Ci-dessous nous nous occupons d'une méthodes d'investigation de tels principes ou tendances.

On a intérêt à suivre le développement des formes et fonctions dans un long espace de temps, de préférence à partir de l'indo-européen commun jusqu'aux langues les plus évoluées, telles que l'anglais ou le français (cf. Gawełko 2001a:40). Le terme »évoluée« veut dire ici »éloignée de la proto-langue«.

Dans cette optique, on pourrait opérer au moins trois coupes horizontales: la première serait représentée par une langue ancienne, le sanskrit par exemple, la deuxième par une langue slave, la troisième par l'anglais ou le français. On sait que les langues slaves se sont éloignées moins de l'indo-européen commun que les langues germaniques ou romanes, en particulier l'anglais. Un inconvénient saute aux yeux: les langues modernes peuvent être comparées sur la base d'un corpus satisfaisant quantitativement et qualitativement, les langues anciennes non. Le corpus défectueux est souvent une source d'erreurs, cf. l'objection faite par Disterheft à tous ses prédécesseurs: »The confusion which is prevalent among previous descriptions of the Rigveda infinitive is consistent: small data collections have led to erroneous descriptions.« (1980: 34) Plus encore que l'ampleur du corpus, c'est sa représentativité insuffisante qui constitue le danger pour le chercheur. Par exemple, Disterheft (1980: 13) parle du passif en avestan en se fondant sur des textes poétiques. La fréquence de cette forme dans la langue parlée pouvait être sensiblement différente.

Les difficultés ne s'arrêtent pas au niveau du corpus, il faut ajouter différents problèmes résolus normalement par les locuteurs natifs, différents problèmes de phonétique, etc.

Étant donné l'insuffisance du champ d'investigation offert par les vieilles langues, il y a tout intérêt à suivre les tendances évolutives des langues accessibles à une analyse multilatérale complexe et se trouvant à des étapes évolutives différentes.

En ce qui concerne les langues modernes, on dispose non seulement de textes nombreux et variés, y compris des textes parallèles, mais aussi de descriptions typologiques de langues particulières (par ex. Albrecht 1970, Criado de Val 1972, Gak 1977, Hawkins 1986, Malblanc 1966, Mańczak 1991, Vinay/Darbelnet 1958, etc.). L'étude des langues modernes peu évoluées et de langues modernes hautement évoluées, fondée sur un corpus représentatif, est à même de compléter l'analyse historique excessivement sommaire des vieilles langues indo-européennes. 
En principe, la réorganisation de la grammaire des langues indo-européennes va dans la même direction dès le début jusqu'à nos jours. Ainsi l'étude des formes et fonctions apparaissant dans les langues modernes se trouvant à différentes étapes évolutives permet de mieux déterminer la spécificité des tendances particulières.

La réorganisation de la grammaire peut être réduite à quelques éléments fondamentaux: $1^{\circ}$ la disparition ou la diminution, $2^{\circ} 1^{\prime}$ apparition ou l'augmentation de $1^{\prime} i m-$ portance de certaines formes ou fonctions.

Pour le premier cas on peut signaler le duel, l'impersonnel ou l'ordre de base OV, pour le second - l'infinitif, le passif ou l'ordre de base VO. Pour l'instant un seul exemple suffit: l'infinitif ne paraît pas avoir existé en indo-européen commun (mais le problème n'est pas définitivement résolu, cf. Jolly 1873:229, García-Ramón 1997:167). Il est attesté dans les vieilles langues. Il augmente sa fréquence jusqu'à l'étape des langues les plus évoluées. Ainsi il présente un emploi modéré dans les langues slaves, il est plus fréquent dans les langues romanes et germaniques.

Cependant la tâche du linguiste consiste non seulement à constater les faits mais aussi à les expliquer. Aujourd'hui l'opinion prédomine que l'explication des changements survenus laissait à désirer tant qu'on négligeait l'importance du passage du type de langue OV > VO (cf. Lehmann 1974: 14).

Prenons l'exemple du passif. En ce qui concerne l'indo-européen commun, nous pouvons accepter $1^{\circ}$ avec Bauer (2000: 25) que 1'opposition actif/passif n'était pas dénotée grammaticalement et $2^{\circ}$ avec Lehmann (1974: 151) que le terme de passivum n'est pas bien fondé vu que les exemples cités n'ont jamais d'agent exprimé. Dans les langues modernes il est dénoté au moyen d'une périphrase qui admet un complément agentif. En ce qui concerne son évolution, il convient de rappeler la constatation de F. Parker: »the change to VO word order acted as a causal agent in the development of the passive « (1976: 449). Cependant, un doute surgit: s'agit-il certainement, chez Parker, d'un lien causal entre les deux phénomènes ou peut-être seulement d'un lien chronologique? Lehmann (1974: 183) constate seulement que ce n'est qu'après le passage de OV à VO que »the central meaning of the middle become that of passive voice «.

Une autre remarque. La constatation de Parker, qui parle du lien entre le développement du passif et le passage de $\mathrm{OV}$ à VO, se rapporte à des langues plus ou moins anciennes. Elle peut éventuellement expliquer la faiblesse du passif des vieilles langues (de type OV) par rapport au passif des langues slaves (de type VO), mais ne dit rien sur la faiblesse relative du passif slave par rapport au passif des langues romanes et plus encore par rapport au passif anglais.

Nous pensons que c'est la tendance analytique qui caractérise les langues indoeuropéennes dès le moment de la proto-langue jusqu'à l'étape des langues les plus évoluées, surtout anglaise et française.

Le premier problème à établir est celui de savoir si en effet les langues indo-européennes s'acheminent vers l'analytisme. Différentes opinions ont été avancées à ce sujet (cf. Lehmann 1974:248, Harris 1978: 119-120, Wright 1983:15, Schwegler 1990:190, etc.). Pour notre part, nous avons essayé à plusieurs reprises de montrer 
que les exemples fournis par différents chercheurs en vue de contester le progrès de l'analytisme dans l'histoire des langues indo-européennes s'expliquent par l'action d'autres tendances, le plus souvent la tendance à l'économie (par ex. Gawełko 2001a: 27-29, 2001b: 12-14). L'existence de différentes tendances n'élimine pas l'importance particulière de la tendance analytique. On peut admettre que dans la langue différentes tendances se manifestent, souvent contradictoires, définies en anglais par des termes tels que conflicting goals. La question reste de savoir quelle est l'importance qu'on devrait assigner à chacune d'elles dans le développement des langues indo-européennes.

La tendance analytique permet d'envisager les trois coupes horizontales envisagées ci-dessus (les vieilles langues - les langues slaves - 1'anglais et le français). Différents critères motivent cette répartition: critère de Greenberg (la quantité des morphèmes divisée par la quantité des mots), contingent des mots dans un texte parallèle, contingent des mots à fonction grammaticale, contingent des sujets pronominaux, contingent des prépositions, contingent des mots auxiliaires, proportion des mots génériques par rapport aux mots spécifiques, etc.

Ainsi, la tendance analytique est un facteur dont le pouvoir explicatif semble être plus grand que celui du passage de OV à VO: il explique, au contraire du passage de $\mathrm{OV}$ à $\mathrm{VO}$, la raison de la faiblesse relative du passif slave (de type $\mathrm{VO}$ ) par rapport au passif anglais (aussi de type VO). Cependant, le même doute persiste pour les deux facteurs: s'agit-il d'un lien causal entre ces facteurs et le développement du passif? On connaît bien la motivation de la fréquence considérable du passif dans les langues dépourvues de déclinaison: dans les langues slaves on dit correctement, comme en pol. Matka kupiła sukienkę »la mère a acheté une robe « et Sukienkę kupiła matka, le sujet et l'objet y étant définis par les désinences. Dans les langues sans déclinaison, on commence la phrase par l'objet en employant un passif ou une dislocation: La robe a été achetée par la mère ou La robe, la mère l'a achetée.

Un argument en faveur du lien causal entre la tendance analytique et le développement de certaines formes et fonctions est apporté peut-être par le fait qu'un certain nombre de ces éléments évolue au fur et à mesure qu'évolue la tendance analytique.

Nous présentons quelques exemples, fondés tantôt sur des travaux déjà publiés tantôt sur un corpus dont les détails sont indiqués ci-dessous.

Le corpus I (presque 400000 mots graphiques pour chaque langue prise en compte: allemande, anglaise, espagnole, française, italienne, polonaise, portugaise et roumaine) se compose de 6 ouvrages entiers ( 4 ouvrages français: $\mathrm{CH}, \mathrm{E}, \mathrm{P}, \mathrm{V}, 1$ ouvrage anglais: A et 1 ouvrage latin: IC), des 3 premiers chapitres d'un ouvrage portugais (ML) et de fragments de deux ouvrages polonais (CD et $\mathrm{Q}$ ). Pour chaque ouvrage, nous disposons de l'original et de traductions en sept langues (pour IC - en huit langues). Une exception: le texte CD n'a pas de version portugaise, ce qui nous a forcé de prendre en compte un fragment de la version portugaise d'un autre ouvrage (BA) aux dimensions équivalentes à celles du texte manquant. Trois de ces ouvrages sont rédigés dans une langue proche de la langue parlée: $\mathrm{A}, \mathrm{ML}$ et $\mathrm{CD}$. La langue de IC est quelque peu archaïsante. 
Le corpus II se compose, pour chaque langue, de fragments des neuf ouvrages indiqués plus haut. De chaque ouvrage, nous avons retenu le fragment initial et le fragment final, chacun comportant, dans la version française, 2500 mots graphiques, à ceci près que nous ne coupons pas les phrases. Le corpus français comporte ainsi 45057 mots graphiques, au lieu de 45000 si les phrases avaient été coupées. Le corpus français une fois établi, nous avons établi aussi le corpus des autres langues qui est à chaque fois composé des fragments équivalents.

En principe, nous opposons le polonais, hautement synthétique, à l'anglais et au français, analytiques. Cependant profitant de notre corpus, nous déterminons également si les différences typologiques secondaires qui caractérisent les cinq langues romanes présentent un rapport avec les tendances évolutives de certaines formes et fonctions.

Les trois exemples qui suivent sont destinés à montrer le rapport entre quelques tendances évolutives et le degré de synthétisme/analytisme de la langue oů elles se manifestent.

L'infinitif. Il provient d'une forme nominale figée (cf. p.ex. Jeffers 1975:135). Cependant, au cours de son évolution, il s'approprie graduellement certaines particularités verbales telles que l'expression de l'aspect (fr. faire - avoir fait, pol. robić - zrobić), de la voix (fr. faire - être fait), la présence d'un objet (fr. lire un livre, cf. il lit un livre). Sporadiquement il peut aussi être accompagné d'un sujet syntaxique au nominatif (ex. prt. Chegou, por fim, o tempo de ela partir para Lisboa. - ML 86). Sa verbalité augmente avec la possibilité de se combiner avec un auxiliaire en formant des temps composés, etc. (cf. aussi Sala, éd., 2001: 277). Un infinitif évolué est un infinitif qui comporte des catégories grammaticales bien développées propres au verbe fini.

Le critère de base c'est le nombre global des infinitifs actifs et passifs établi dans notre corpus II: 1 prt. 1512 occurrences, 2 esp. 1441, it. 1431, fr. 1404, 3 ang. 1105, 4 all. 1018,5 pol. 903,6 roum. 185. Conformément à la pratique courante, ces données ne contiennent pas l'infinitif formant le futur et le conditionnel.

Les infinitifs passifs sont beaucoup plus rares que les actifs. Leurs occurrences ont été établies sur la base du corpus I, presque 10 fois plus grand que le corpus II: 758 exemples anglais, 280 allemands, 276 français, 269 portugais, 263 italiens, 176 espagnols, 101 polonais et 91 roumains. Ces chiffres présentent un certain rapport avec la vitalité du passif dans ces langues (cf. Gawełko 1999: 14).

Un exemple tiré de IC:

lat. De ardenti amore et vehementi affectv svscipiendi Christvm. - fr. Du désir ardent de recevoir Jésus -Christ. - prt. Do grande e ardente desejo de receber a Jesus Cristo. esp. Abrasado amor y vivas ansias de recibir a Cristo. - it. L'ardente amore e l'intenso desiderio di ricevere Cristo. - roum. Iubirea fierbinte şi dorul mare de a primi pe Hristos. - ang. On Ardent Love and Eager Desire to Receive Christ. - all. Die brennende Liebe und starke Sehnsucht, Christus zu empfangen. - pol. Żarliwa mitość i gwattowne pożqdanie przyjecia Chrystusa. Seuls le latin et le polonais n'emploient pas l'infinitif.

Il y a deux conclusions à tirer.

En premier lieu, les grandes différences typologiques qui se manifestent entre le 
polonais et l'anglais ou le français présentent un rapport étroit avec la vitalité de l'infinitif: celui-ci est beaucoup plus faible en polonais que dans ces deux langues. On peut dire plus: il est beaucoup plus faible en polonais que dans n'importe quelle langue romane et, paraitt-il, aussi dans n'importe quelle langue germanique.

En deuxième lieu, les différences limitées qui se manifestent dans le degré de synthétisme/analytisme caractérisant les langues romanes présentent un rapport lâche avec la vitalité de l'infinitif. Ce n'est pas le français, le plus analytique, mais le portugais qui jouit de la plus grande vitalité de son infinitif. Ce fait confirme l'importance de facteurs secondaires.

On peut tenter d'expliquer la position de l'infinitif français en rappelant que la marche du français vers l'analytisme, comme celle de n'importe quelle langue romane, n'est pas homogène: il se sépare des langues sœurs dans certaines des catégories, tandis que dans d'autres il reste une langue typiquement romane.

L'infinitif portugais non seulement s'avère le plus fréquent mais aussi il subit le plus la tendance à la grammaticalisation propre à l'infinitif indo-européen: il prend relativement souvent un sujet au nominatif (cf. Gawełko 2005), qui est plus rare dans les autres langues romanes, et très rare ou inexistant dans les autres langues. L'infinitif portugais prend aussi des désinences, ce qui est un phénomène très rare. Par contre, la dernière étape du développement de l'infinitif indo-européen: la substantivation au moyen d'un article n'y est pas aussi bien représentée qu'en italien (cf. Gawełko 2004).

Bref, les données ci-dessus confirment notre thèse de cette façon que l'infinitif polonais s'avère beaucoup plus pauvre que l'infinitif de n'importe quelle langue romane (le roumain excepté; son appartenance à l'union des langues balkaniques explique suffisamment bien la grande faiblesse de son infinitif) ou germanique. L'infinitif polonais ne prend pas un sujet au nominatif, il n'est substantivé qu'en exerçant une fonction, celle de sujet par exemple. Par contre les différences typologiques limitées entre les langues romanes ont un rapport plutôt lâche avec le degré de développement de l'infinitif. Bien entendu, dans les vieilles langues l'infinitif était beaucoup plus faible que l'infinitif slave: provenant d'une forme nominale figée il était en train de devenir infinitif.

Le passif du type: être + participe. Nous rappelons des données d'après notre travail sur le passif roman (Gawełko 1999: 14): ang. 1301 occurrences, all. 928, fr. 785, roum. 776 , it. 671 , prt. 626 , esp. 514 , pol. 426 . Ces chiffres ainsi que les constatations faites par Bauer, Lehmann et Parker, rappelées plus haut, conduisent à quelques conclusions générales. Le passif n'a pas été exprimé grammaticalement en indo-européen commun. Son développement a été accéléré avec le passage du type de langue OV au type VO. Il est donc plus développé dans les langues slaves, de type VO, que dans les vieilles langues.

Dans les langues romanes et germaniques il augmente sensiblement sa fréquence par rapport au passif slave. La position privilégiée du passif anglais ainsi que la position du passif français s'expliquent par l'analytisme de ces langues. C'est surtout la position de l'allemand qui devrait être expliquée. Une constatation analogue à celle 
faite au paragraphe précédent: les différences typologiques relativement secondaires ont un rapport plutôt lâche avec la fréquence du passif.

Un exemple:

fr. On lui avait fait un lit dans la pièce commune. (P, p. 167) - prt. Tinham-lhe feito uma cama no compartimento comum. - esp. Le hicieron una cama en la habitación común. - it. Gli avevano fatto un letto nella stanza in comune. - roum. Î făcuseră un pat în odaia comună. - ang. He was given a bed in the living-room. - all. Im gemeinsamen Aufenthaltsraum war ihm ein Bett aufgeschlagen worden. - pol. We wspólnym pokoju zrobiono mu postanie. Seules les langues germaniques emploient un passif.

L'impersonnel. Il a existé dans la proto-langue. Il apporte un argument en faveur de la thèse que celle-ci était, à une époque ancienne, une langue de type actif (cf. Bauer 2000: 335). Cependant, au cours de l'évolution des langues indo-européennes, il diminue son importance. L'argument connu en faveur de cette opinion est que, dans les langues analytiques, le modèle phrastique de base SVO connaît de moins en moins d'exceptions. Un autre argument est qu'on a relevé pour certaines langues une ou des époques où une grande quantité d'impersonnels ont été remplacés par des formes personnelles (pour l'anglais, on se fonde en général sur van der Gaaf, par ex. Moessner 1984). Un autre argument est apporté par des indo-européistes. Ainsi, B. Bauer constate: »Impersonal verbs are found in all Indo-European languages; the more one goes back in time, the more one will find.« (Bauer 2000: 97).

Un exemple type:

fr. J'avais chaud sous mes vêtements sombres. (E, p. 26) - ang. I was hot under my dark clothes. - all. Ich schwitzte in meinem dunklen Zeug. - esp. Tenía calor con mi traje oscuro. - it. Avevo caldo sotto i miei vestiti scuri. - prt. Tinha calor, com o meu fato escuro. - roum. Îmi era cald în hainele mele de culoare închisă - pol. W ciemnym ubraniu było mi goraco. Seuls le roumain et le polonais emploient un impersonnel.

À la rigueur, nous pourrions déjà tirer une conclusion à partir de ces remarques, qui serait de nature à appuyer notre thèse. Cependant notre corpus I est susceptible d'apporter une certaine contribution à la détermination de la vitalité de l'impersonnel. Nous présentons les constructions du type être + prédicat adjectival/adverbial qui ne se rapportent pas aux phénomènes atmosphériques, comme dans ang. It is so good to be with Him! (ML). Le corpus offre 184 exemples français et 206 exemples polonais. L'analyse de caractères spécifiques des constructions en cause augmente quelque peu la différence entre les deux langues. Une autre enquête fondée sur la moitié de notre corpus II (il embrasse les fragments des ouvrages $\mathrm{CD}, \mathrm{CH}, \mathrm{E}$ et $\mathrm{ML}$ ) a permis de relever la totalité des occurrences de l'impersonnel en ang. it, there (non pas en ang. one, roum. se, pol. się) comme suit: en polonais 106 occurrences, en roumain 85 et en anglais 51. Il y a donc une différence modérée entre le polonais et le roumain et une différence nette entre les deux et l'anglais. Néanmoins une remarque s'impose: la différence entre la langue slave et les autres prises en compte, sensiblement plus analytiques, n'est pas aussi nette que c'est le cas de l'infinitif et celui du passif. La raison 
en réside, paraît-il, dans l'économie de l'impersonnel et dans la simplicité des situations météorologiques pour lesquelles l'impersonnel est particulièrement caractéristique: il pleut, plus économique que la pluie tombe, n'apporte pas moins d'informations; la nature de la pluie est de tomber.

CONCLUSION. Pour des raisons évidentes, il est beaucoup plus facile d'étudier les langues modernes que les vieilles langues indo-européennes. Comme les langues modernes se trouvent à différentes étapes de leur évolution, qui va en principe dans le même sens, il est possible de prendre en compte, dans une analyse des formes et fonctions indo-européennes, des langues modernes se trouvant à différentes étapes de leur évolution. Ainsi, pour examiner les tendances évolutives des formes telles que l'infinitif, le passif ou l'impersonnel, on a intérêt à faire quelques coupes horizontales, par exemple: les vieilles langues - les langues slaves - les langues anglaise et française, la première étant le moins, la dernière le plus éloignée de la proto-langue.

S'il s'agit de l'évolution des trois formes, l'étape des langues slaves est toujours intermédiaire: l'impersonnel est le plus fort dans les vieilles langues et le plus faible en anglais et en français, tandis que l'infinitif et le passif sont les plus faibles dans les vieilles langues et les plus forts en anglais et en français.

Les différences typologiques secondaires qui caractérisent les langues romanes, telles que le degré de synthétisme/analytisme, présentent un rapport plutôt lâche avec le degré de développement de ces formes. 


\section{Bibliographie}

ALBRECHT Jürgen, 1970, Le français langue abstraite?, Tübingen.

BAUER Brigitte, 2000, Archaic Syntax in Indo-European, Berlin - New York: Mouton de Gruyter.

CRIADO DE VAL Manuel, 1972, Fisonomía del español y de las lenguas modernas, Madrid: Editorial S.A.E.T.A.

DisterheFt Dorothy, 1980, The Syntactic Development of the Infinitive in Indo-European, Columbus, Ohio: Slavica Publishers.

GAK Vladimir, 1977, Sopostavitel'naja leksikologija, Moscou: Meždunarodnye otnošenija.

GaRCíA-RAMÓn José Luis, 1977, Infinitive im Indogermanischen? Zur Typologie der Infinitivbildungen und zu ihrer Entwicklung in den älteren indogermanischen Sprachen, Incontri Linguistici 20, 45-69.

GAWEŁKo Marek, 1999, L'étude sur l'ordre des mots dans les langues romanes, vol. 2: La passivation, Lublin: Towarzystwo Naukowe KUL.

GAWELKO Marek, 2001a, Essai de classification fonctionnelle des langues romanes, Romance Philology 55, 21-40.

GAWEŁKO Marek, 2001b, Język polski wobec tendecji analitycznej języków indoeuropejskich, Polonica XXI, 11-24.

GAWEŁKO Marek, 2004, Quelques particularités de l'infinitif substantivé en espagnol et en portugais, Bulletin Hispanique 2, 615-635.

GAWEŁKO Marek, 2005, 2006, 7-23, Le sujet syntaxique de l'infinitif en espagol et en portugais, Verba 32.

HARRIS Martin B., 1978, The Evolution of French Syntax, Londres: Longman.

HASPELMATH Martin, 1999, Are there principles of grammatical change?, Journal of Linguistics 35, 579-595.

HAWKINS John A., 1986, A Comparative Typology of English and German, Londres: Croom Helm.

JEFFERS Robert J., 1975, Remarks on Indo-European infinitives, Language 51, 1, 133-148.

JoLly Julius, 1873, Geschichte des Infinitivs im Indogermanischen, Munich: Theodor Ackermann.

Lehmann Winfred O., 1974, Proto-Indo-European Syntax. Austin - Londres: University of Texas Press.

MALBLANC Alfred, 1966, Stylistique comparée du français et de l'allemand, Paris: Didier.

MANCZAK Witold, 1991, La classification des langues romanes, Cracovie: Universitas.

Moessner Lilo, 1984, Impersonal Constructions in Early Middle English, Studia Anglica Posnaniensia 17, 29-38.

PARKER Frank, 1976, Language change and the passive voice, Language 52, 2, 449-460.

Sala Marius (éd.), 2001, Enciclopedia limbii române, Bucarest: Univers Enciclopedic.

SCHWEgler Armin, 1990, Analycity and Syntheticity. A Diachronic Perspective with Special Reference to Romance Languages, Berlin-New York: Mouton de Gruyter.

VINAY J.P. \& Darbelnet J., 1958, Stylistique comparée du français et de l'anglais, Paris: Didier.

WRIGHT Roger, 1983, Unity and Diversity among the Romance Languages, Transactions of the Philological Society: 1-22.

${ }^{1}$ Camus, A., L'étranger, Paris, Gallimard, 1942; El extranjero, Madrid, El Libro de Bolsillo, 1982; Lo straniero, Milan, Tascabili Bompiani, 1988; O estrangeiro, Lisbonne, Unibolso, s.d.; Străinul, in Străinul, Ciuma, Căderea, Exilul şi împărăția, Bucarest, RAO International Publishing Company, p. 27-92, 1993; The Outsider, Londres, Penguin Books, 1983; Der Fremde, Reinbeck bei Hambourg, Rowohlt, 1993; Obcy, Varsovie, Krąg, 1991. (E)

2 Camus, A., La chute, Paris, Gallimard, 1989; La caída, Madrid, Alianza Editorial, 1982; La caduta, Milan, Tascabili Bompiani, 1989; A Queda, Lisbonne, Livros do Brasil e Editorial Verbo, 1971; Căderea, in Străinul, Ciuma, Căderea, Exilul şi împărăția, Bucarest, RAO International Publishing Company, p. 295-369, 1993; The Fall, Londres, Penguin Books, 1963; Der Fall, Reinbeck bei Hambourg, Rowohlt, 1995; Upadek, Varsovie, Krąg, 1991. (CH)

3 Camus, A., La peste, Paris, Gallimard, 1989; A peste, Lisbonne, Livros do Brasil, s.d.; La peste, Barcelone, Edhasa, 1977; La peste, Milan, Tascabili Bompiani, 1989; Ciuma, in Străinul, Ciuma, Căderea, Exilul şi împărăția, Bucarest, RAO International Publishing Company, p. 93-294, 1993; The plague, Londres, Penguin Books, 1960; Die Pest, Hambourg, Rowohlt, 1994; Dżuma, Varsovie, Jota, 1991. (P)

${ }^{4}$ Maupassant, G. de, Une vie, Paris, Gallimard, 1974; Una vida, in G. de Maupassant, Obras completas, p. 149285, Madrid, Aguilar, 1965; Uma vida, Mem Martins, Livros de bolso Europa-America, 1974; Una vita, Farigliano, Arnoldo Mondadori Editore, 1984; O viaţă, Chişinău, Făt-Frumos, 1994; A Woman's Life, Londres, Penguin Books, 1965; Ein Leben, Frankfurt/M-Berlin, Ullstein, 1995; Historia jednego życia, Varsovie, Zrzeszenie Księgarstwa, 1985. (V)

5 Memórias da Irmã Lúcia, Fátima, Postulação, 1978; Mémoires de Sceur Lucie, Fátima, Postulação, 1980; Memorias de la Hermana Lucía, Fátima, Postulação, 1978; Memorie di Suor Lucia, Fátima, Postulação, 1980; Sora Lucia despre Fatima, Fátima, Secretariado dos Pastorinhos, 2002; Fatima in Lucia's own words, Fátima, Postulação, 1976; Schwester Lucia spricht über Fátima, Fátima, 1975; Siostra Eucja mówi o Fatimie, Fátima, Postulaçăo, 1978 (jusqu'à la p. 104 de l'original portugais). (ML) 
6 Tomas a Kempis, De imitatione Christi; ( $O$ naśladowaniu Jezusa Chrystusa), édition bilingue, Varsovie, Instytut Wydawniczy Pax, 1981; L'imitation de Jésus-Christ, Paris, Édition du Seuil, 1961; L'imitazione di Cristo, Milan, Edizioni Paoline, 1988; Imitación de Cristo, Barcelone, Editorial Regina, 1987; Imitação de Cristo, Braga, Editorial Franciscana, 1980; Urmarea lui Hristos, Timişoara, Editura Metropoliei Banatului, 1991; The Imitation of Christ, Londres, Penguin Books, 1965; Thomas von Kempen, Die Nachfolge Christi, Kevelaer, Butzon \& Bercker, 1990; Tomasz à Kempis, Naśladowanie Chrystusa, Cracovie, WAM, 2000. (IC)

7 Carrol, L., Alice's adventures in Wonderland - Les aventures d'Alice au Pays des merveilles, Paris, AubierFlammarion, 1970; Aventuras de Alicia en el país de las maravillas, Barcelone, Moby Dick, 1973; Alice nel Paese delle Meraviglie, in Alice nel Paese delle Meraviglie, Attraverso lo specchio, Milan, Garzanti, p. 1-141, 1989; Alice no País das Maravilhas, Mem Martins, Publicações Europa-America, 1998; Alisa în ţara minunilor, Craiova, Editura Literatorul, 1991; Alice im Wunderland, Hambourg, Cecilie Dressler Verlag, 1990; Przygody Alicji w Krainie Czarów, Varsovie, Lettrex, 1990. (A)

${ }^{8}$ Sienkiewicz, H., Quo Vadis, Varsovie, Labos, 1990; Quo vadis, Paris, Le livre de poche 3161, 1971; Quo vadis? Madrid, Aguilar, 1967; Quo vadis?, Milan, Biblioteca Universale Rizzoli, 1984; Quo Vadis?, Mem Martins, Livros de bolso Europa-America, 1974; Quo Vadis, Bucarest, Editura Universul, 1945; Quo Vadis?, Sawtry-New York, Hippocrene-Dedalus, 1993; Quo Vadis, Zurich, Diogenes, 1985 (chap. X-XII du premier volume). (Q)

${ }^{9}$ Andrzejewski, J., Popiót i diament, Varsovie, Krajowa Agencja Wydawnicza, 1966; Cendres et diamant, Paris, Gallimard, 1967; Cenizas y diamantes, Barcelone, Luis de Caralt, 1966; Cenere e diamanti, Lerici Editori, Milan, 1961; Cenuşă şi diamant, Bucarest, Editura pentru Literatură Universală, 1968; Ashes and Diamond, Eveston, Northwestern Univ. Press, 1991; Asche und Diamant, Frankfurt/Main, Suhrkampf Verlag, 1984 (chap. IV et $\mathrm{V})(\mathrm{CD})$

10 Maupassant, G. de, Bel-ami, Livros de bolso Europa-América, Guimarães Editores, 1972. (BA) 


\section{Povzetek}

\section{RAZVOJNI VIDIK V INDOEVROPSKIH JEZIKIH}

Prispevek želi z razvojnega vidika osvetliti nekaj slovničnih enot v sicer tipološko različnih indoevropskih jezikih. Gledé na skladenjsko rabo enot kot so nedoločnik, trpnik ali brezosebni glagol je mogoče razločiti vsaj tri razvojne stopnje: najstarejšo, kot jo predstavlja sanskrt; srednjo, vmesno, kot jo kažejo moderni indoevropski jeziki, ki se od osnove, torej od prvotnega jezika niso preveč oddaljili, tako kot na primer slovanski; moderno razvojno stopnjo, kar kažejo jeziki, ki so v prvotni jezik vnesli močne inovacije, kot na primer francoščina ali angleščina. Primerjavo med drugo in tretjo razvojno stopnjo je $z$ diahronim pogledom mogoče zasnovati na obsežnem korpusu raznolikih besedil in seveda tudi na velikem številu tehtnih razprav o navedenih skladenjskih vprašanjih, kar dovoljuje izostriti o teh celostno študijo od najstarejše dobe do današnje razvojne stopnje. 